\title{
Classificação multivariada de curvas de progresso da requeima do tomateiro entre acessos do Banco de Germaplasma de Hortaliças da UFV
}

\author{
Multivariate Classification of the progress curves of tomato late blight among accessions \\ from the UFV Germoplasma Vegetable Bank
}

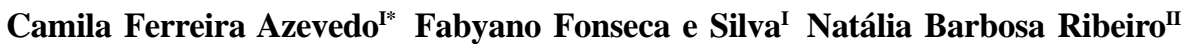 \\ Derly Jose Henriques da SilvaII Paulo Roberto Cecon ${ }^{\mathrm{I}}$ \\ Leiri Daiane Barili" ${ }^{I}$ Valeria Rosado Pinheiro ${ }^{I}$
}

\section{- NOTA-}

\section{RESUMO}

O objetivo deste trabalho foi apresentar uma metodologia de análise de experimentos em fitopatologia que considera a comparação de curvas de progressos de doenças na presença de um grande número de tratamentos por meio da análise de cluster. Foram cultivados 42 acessos do Banco de Germoplasma de Hortaliças (BGH) da Universidade Federal de Viçosa (UFV). Ajustou-se o modelo exponencial aos dados de percentagem de severidade de requeima, e as estimativas obtidas quanto à incidência inicial da doença $\left(y_{o}\right)$ e taxa de progresso da doença (r) foram submetidas à análise de variância multivariada (Manova), seguindo o delineamento de blocos casualizados. As médias ajustadas foram submetidas à análise de agrupamento hierárquico, o método centroide. Observou-se um número ótimo de seis grupos distintos.

Palavras-chave: análise de cluster, melhoramento genético.

\section{ABSTRACT}

The objective of this paper was to present a methodology for the analysis of experiments in plant pathology that considers the comparison of disease progress curves in the presence of a large number of treatments by cluster analysis. Forty-two accessions were grown from the Germoplasma Vegetable Bank (BGH), of Universidade Federal de Viçosa (UFV). The exponential model was fitted to the data of late blight severity percentage, and the obtained parameter estimates obtained on the initial incidence of the disease $\left(y_{0}\right)$ and rate of disease progression ( $r$ ) - were submitted to the multivariate analysis of variance (MANOVA). The adjusted means were submitted to the cluster analysis. An optimal number of six distinct groups was observed.

Key words: cluester analysis, plant breeding.
A família Solanaceae é de grande importância para a agricultura, destacando-se o tomate Lycopersicon esculentum (Mill) por sua versatilidade comercial. Contudo, os agricultores encontram muitas dificuldades devido à susceptibilidade da cultura a diversas doenças e pragas. Isso ocorre, pois o tomate, produto de sucessivas seleções, teve sua variabilidade genética restringida (LABORY et al., 1999).

O fungo Phytophthora infestans, agente etiológico da requeima, causa grandes perdas nos campos de cultivo, principalmente quando há alta umidade e baixa temperatura. O uso de fungicidas é a principal medida de controle da doença, elevando os custos de produção. Dessa forma, torna-se necessário o conhecimento das possíveis fontes de resistência do tomate (SUINAGA et al., 2003), as quais podem ser encontradas nas espécies silvestres registradas nos bancos de germoplasma.

Atualmente, o BGH-UFV (Banco de Germoplasma de Hortaliças da UFV, www.ufv.br/bgh) possui 7225 acessos, com 25 famílias e 106 espécies, tendo maior representação a família Solanácea, pois, dentre estas, 870 acessos são do gênero Lycopersicon. Uma forma de avaliar o comportamento da requeima do tomateiro é por meio do ajuste de modelos de progresso

IDepartamento de Estatística, Centro de Ciências Exatas (CCE), Universidade Federal de Viçosa (UFV), Avenida P.H. Rolfs, s/nº, 36570-000, Viçosa, MG, Brasil. Email: camila.azevedo@ufv.br.*Autor para correspondência.

"Departamento de Fitotecnia, Centro de Ciências Agrárias (CCA), UFV, Viçosa, MG, Brasil. 
de doenças (CAMPBELL \& MADEN, 1990; FIORINI et al., 2010), cujos parâmetros apresentam interpretação biológica. Quando o pesquisador dispõe de vários tratamentos, é usual comparar a forma da curva de progresso da doença entre estes, sendo tal comparação realizada para as estimativas dos parâmetros dos modelos. Quando o número de tratamentos é elevado, alguns métodos tradicionais que realizam comparações duas a duas como os descritos por CAMPBELL \& MADEN (1990) e REGAZZI \& SILVA(2004), tornam-se inviáveis, devido ao número total de comparações. De acordo com KHATTREE \& NAIK (2000), a utilização da técnica multivariada de agrupamento apresenta-se como uma solução para este problema, uma vez que tende a agrupar tratamento com vetores de médias que caracterizam similaridade entre as curvas, assumindo ou não um dado nível confiança.

O objetivo do presente trabalho é apresentar uma metodologia de análise de experimentos em fitopatologia que considera a comparação de curvas de progressos de doenças na presença de um grande número de tratamentos ( $>40$ ) por meio da análise de cluster. Tal metodologia avalia o desenvolvimento da requeima do tomateiro em relação a diversos acessos do BGH-UFV. Com esse propósito, um experimento foi conduzido no Campo Experimental do Departamento de Fitotecnia da UFV, no inverno de 2005. Os acessos do BGH foram transplantados para o campo 30 dias após a semeadura. O delineamento experimental utilizado foi o de blocos casualizados com três repetições. Foram cultivados 42 acessos do BGH, sendo realizados todos os tratos culturais recomendados para a cultura.

O inóculo obtido foi resultado da mistura de esporângios, cuja concentração foi ajustada com o hemacitômetro para $1 \times 10^{3}$ esporângios $\mathrm{mL}^{-1}$. A inoculação foi realizada 60 dias após o transplantio e as avaliações foram feitas a cada três dias, sendo a característica avaliada a área foliar lesionada pelo fungo $\boldsymbol{P}$. infestans. Essa área foi transformada na variável severidade por meio da utilização do software Severity Pro 1.0. Ao final do experimento, foram obtidos dados de seis avaliações temporais para cada BHG considerado.

Para descrever o comportamento da severidade (y) em função do tempo, além do modelo exponencial (CAMPBELL \& MADEN, 1990) dado por $\mathrm{y}=\mathrm{y}_{\mathrm{o}} \exp (\mathrm{rx})$ ), em que $\mathrm{y}_{\mathrm{o}}$ é a quantidade inicial de doença e $r$ a taxa de progresso, ajustaram-se também modelos mais complexos, tais como Gompertz, Logístico, Brody e Richards (SILVEIRA et al., 2011). Esses ajustes foram realizados para cada repetição de cada BGH mediante o PROC MODEL do software SAS ${ }^{\circledR}$ (SAS,1996) e o modelo Exponencial mostrou-se mais adequado (Tabela 1). Apesar de os avaliadores R2aj e AIC apresentarem desempenho semelhante aos outros modelos, há a vantagem de apresentar maior \% de convergência, a qual implica um maior número de curvas a serem avaliadas mediante métodos multivariados utilizados.

Realizou-se uma análise de variância multivariada utilizando o PROC GLM (SAS, 1996) com a opção MANOVA, a fim de avaliar o efeito dos acessos do BGH sob estas estimativas dos parâmetros do modelo Exponencial por meio do critério de Roy (KHATTREE \& NAIK, 2000). Sendo constatada essa influência (rejeição da hipótese Ho: pelo menos um vetor de médias de estimativas de parâmetros provenientes de um dado tratamento difere-se dos demais), extraíram-se as médias ajustadas por quadrados mínimos (LSMEANS) e as submeteram à análise de agrupamento no PROC CLUSTER (SAS, 1996) pelo método centroide. Este define a coordenada de cada grupo (centroide) como sendo a média das coordenadas de seus objetos e, dessa forma, a distância entre os grupos é a distância entre os centroides. Assim,

Tabela 1 - Modelos utilizados para descrever curvas de progresso da requeima do tomateiro e respectivos avaliadores de qualidade de ajuste.

\begin{tabular}{|c|c|c|c|c|}
\hline \multirow{2}{*}{ Modelo } & \multirow{2}{*}{ № Parâmetros } & \multicolumn{3}{|c|}{---Avaliadores da Qualidade de Ajuste- } \\
\hline & & $\mathrm{R}_{\text {aj }}^{2}$ & AIC & \%Conv. \\
\hline Exponencial & 2 & 0,9807 & 27,0426 & $100 \%$ \\
\hline Richards & 4 & 0,9825 & 35,5717 & 0,1708 \\
\hline Gompertz & 3 & 0,9734 & 30,6113 & 0,8125 \\
\hline Logístico & 3 & 0,9762 & 28,6127 & 0,8583 \\
\hline Brody & 3 & 0,9840 & 27,2852 & 0,9733 \\
\hline
\end{tabular}


a fim de identificar o número ótimo de cluster, foram obtidos os valores dos desvios-padrão da raiz do quadrado médio (RMSSTD), que permite a identificação do número ótimo de cluster por meio do ajuste de um modelo de máxima curvatura. Além disso, utilizou-se o PROC TREE (SAS, 1996) para visualizar o dendograma e verificar quais acessos pertencem aos diferentes grupos obtidos pela discriminação estatística (KHATTREE \& NAIK, 2000). Os códigos do programa utilizado neste artigo poderão ser encontrados no site do Departamento de Estatística (http://www.det.ufv.br).

$\mathrm{Na}$ Manova, foi constatado efeito significativo $(\mathrm{P}>\mathrm{F}=0,0455)$ dos acessos de $\mathrm{BGH}$ sobre o vetor dos parâmetros $\mathrm{y}_{\mathrm{o}}$ e r, sugerindo que diferentes acessos providenciam diferentes curvas. Resultados semelhantes foram obtidos por ABREU et al. (2005), os quais relatam que existe diferença entre estes acessos em relação a área abaixo da curva de progresso.
O número ótimo de cluster foi determinado geometricamente de acordo com LARSON et al. (1998) e CECON et al. (2008). As análises gráficas representadas por este método são apresentadas na figura 1 .

De acordo com a figura 2, nota-se que os acessos do $\mathrm{BGH}$ podem ser agrupados em seis diferentes grupos, de acordo com os vetores de $\mathrm{y}_{\mathrm{o}} \mathrm{e} \mathrm{r}$. Esses resultados são úteis para que se tenha conhecimento dos recursos dos acessos do BGH, sendo possível compará-los e predizer suas possíveis contribuições para futuros programas de melhoramento genético.

A metodologia utilizada possibilitou comparar curvas de progressos de doenças quando se tem um grande número de tratamentos, neste caso 42 acessos do BGH-UFV. A análise de cluster gerou seis grupos que representam as curvas estatisticamente diferentes.

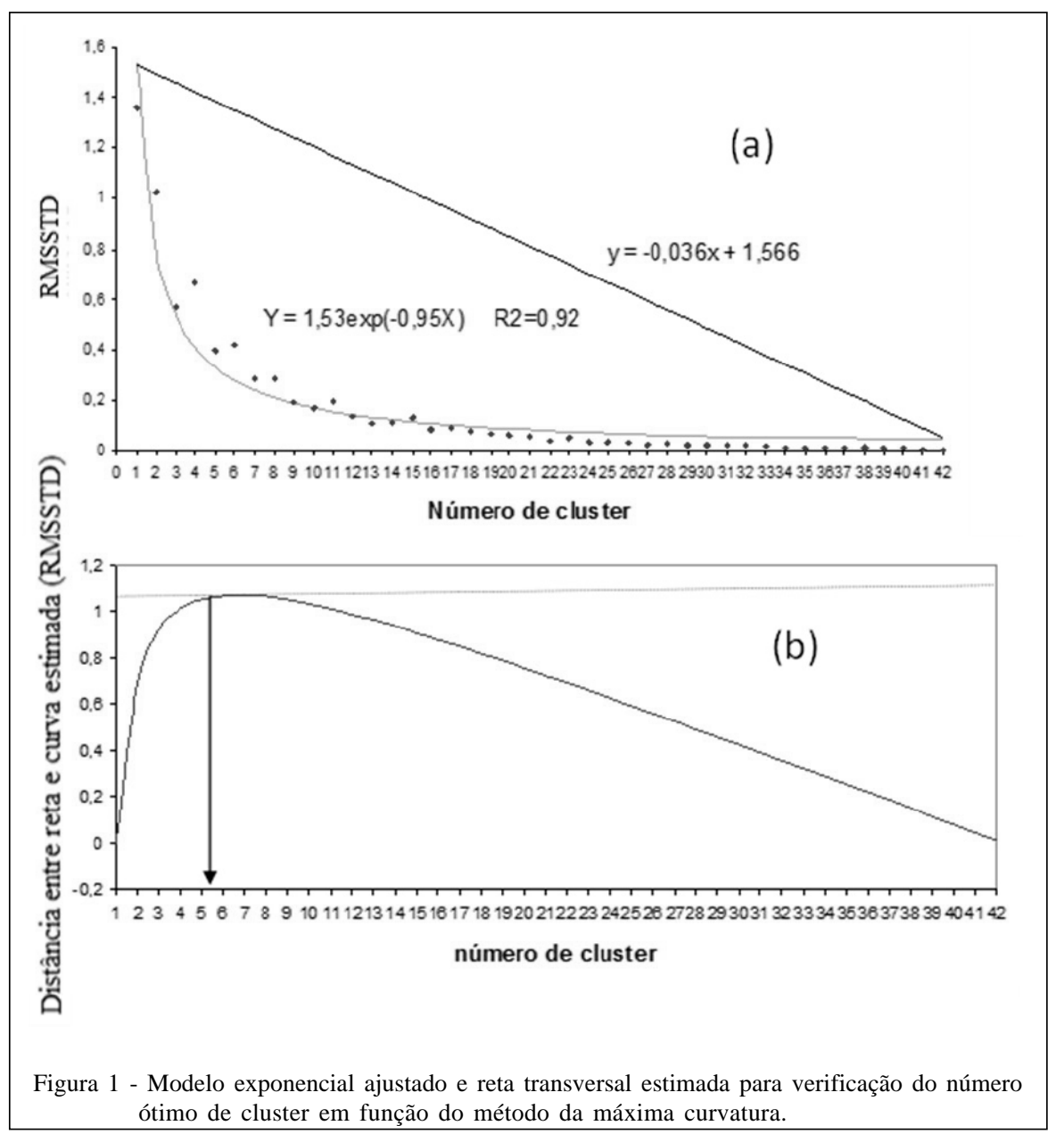

Ciência Rural, v.42, n.3, mar, 2012. 


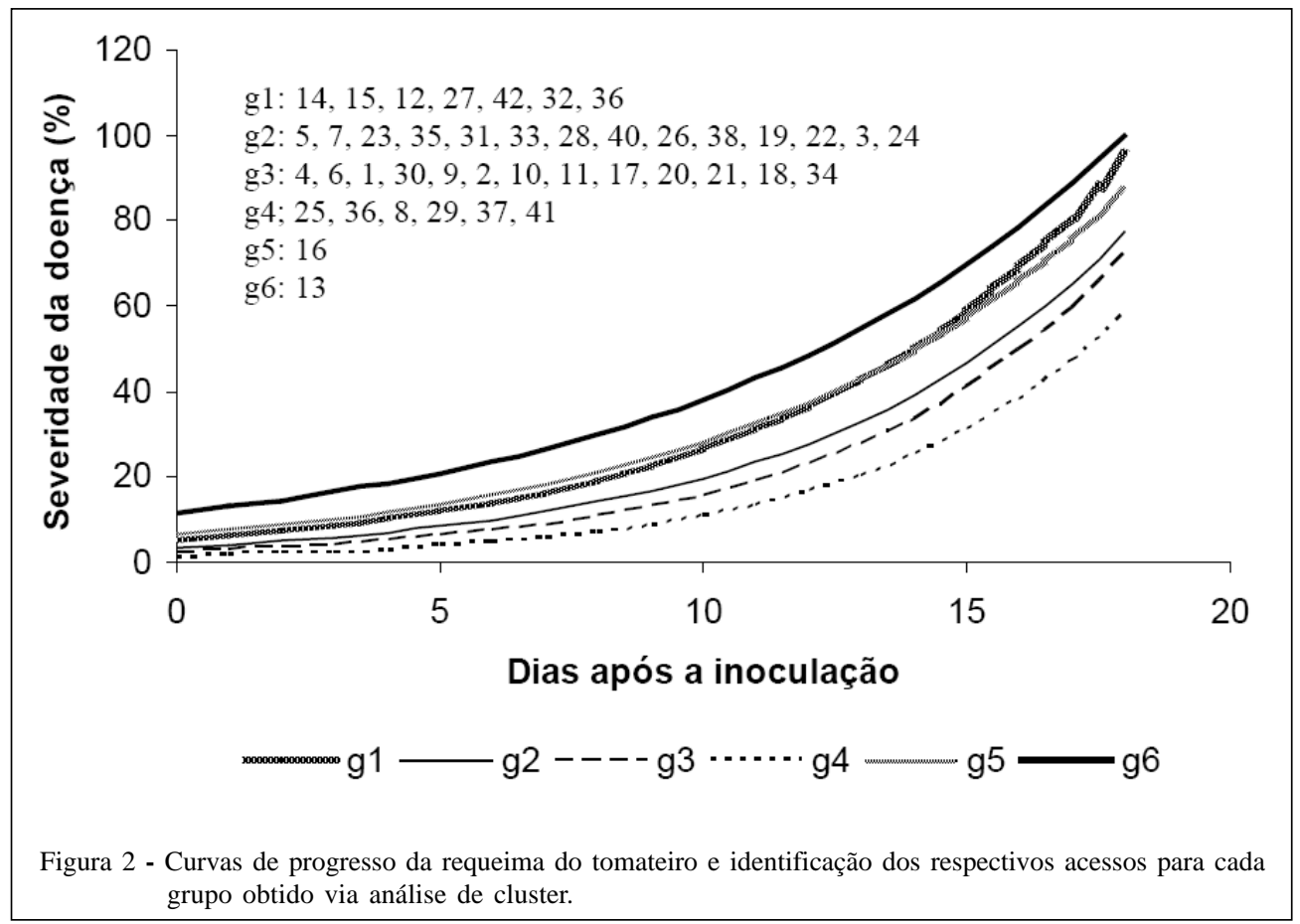

\section{REFERÊNCIAS}

ABREU, F.B. et al. Comparação entre seleção combinada e seleção entre e dentro de famílias F5 para resistência à requeima em tomateiro. In: CONGRESSO BRASILEIRO DE OLERICULTURA, 45.,2005, Fortaleza/CE. Horticultura Brasileira: Resumos... Fortaleza: Embrapa Agroindústria Tropical, 2005. V.23.

CAMPBELL, C.L. et al. Introduction to plant disease epidemiology. New York Wiley-Interscience Publication, John Wiley \& Sons, 1990. 531.

CECON, P.R. et al. Análise de medidas repetidas na avaliação de clones de café 'Conilon'. Pesquisa Agropecuária Brasileira, Brasília, v.43, n.9, p.1171-1176, 2008. Disponível em : <http://dx.doi.org/10.1590/S0100-204X2008000900011>. Acesso em: 20 fev. 2011. doi: 10.1590/S0100204X2008000900011.

FIORINI, C.V.A et al. Agrupamento de curvas de progresso de requeima, em tomateiro originado de cruzamento interespecífico. Pesquisa Agropecuária Brasileira, Brasília, v.45, n.10,p.1095-1101,2010. Disponível em:<http:// dx.doi.org/10.1590/S0100-204X2010001000007>. Acesso em: 10 jan. 2011. doi: 10.1590/S0100-204X2010001000007.

KHATTREE, R.; NAIK, D.N. Multivariate data reduction and discrimination with SAS software. New York: BBU and John Wiley Sons, 2000. 574p.

LABORY, C.R.G. et al. Seleção indireta para teores de 2tridecanona em tomateiros segregantes e sua relação com a resistência à traça do tomateiro. Pesquisa Agropecuária Brasileira, Brasília, v.34, p.723-739, 1999. Disponível em:<http://dx.doi.org/10.1590/0100-204X1999000500002>. Acesso em: 13 dez. 2010. doi: 10.1590/S0100204X1999000500002.

REGAZZI, A.J.; SILVA, C.H.O. Teste para verificar a igualdade de parâmetros e a identidade demodelos de regressão nãolinear.I.dados no delineamento inteiramente casualizado.Revista Matemática e Estatística,São Paulo, v.22, n.3, p.33-45, 2004. Disponível em: <http://jaguar. fcav.unesp.br/RME/fasciculos/v22/ v22_n3/A3_Adair.pdf>. Acesso em:14mai. 2011.

SAS. Statistical Analysis System Institute. SAS/STAT. User's guide, version 6.11. 4.ed. Cary, 1996. V.2, 842p.

SILVEIRA, F.G. et al. Análise de agrupamento na seleção de modelos de regressão não-lineares para curvas de crescimento de ovinos cruzados. Ciência Rural, Santa Maria, v.41, p.692698, 2011. Disponível em: <http://dx.doi.org/10.1590/S010384782011000400024>. Acesso em: 18 mai. 2011. doi: 10.1590/ S0103-84782011000400024.

SUINAGA, F.A. et al. Dissimilaridade genética de fontes de resistência de Lycopersicon spp. a Tuta absoluta (Meyrick, 1917) (Lepidoptera: Gelechiidae). Revista Brasileira de Agrociência, Pelotas, v.9, n.4, p.371-376, 2003. Disponível em: <http://www.ufpel.tche.br/faem/agrociencia/v9n4/ artigo11.pdf $>$. Acesso em: 11 fev. 2011. 